\title{
FGFR3 mutations in prostate cancer: association with low-grade tumors
}

\author{
Silvia Hernández ${ }^{1}$, Silvia de Muga $^{2,3}$, Laia Agell $^{2,3}$, Nuria Juanpere ${ }^{2,3}$, Raquel Esgueva ${ }^{2,3}$ \\ José A Lorente ${ }^{3,4}$, Sergi Mojal ${ }^{5}$, Sergio Serrano ${ }^{2,3}$ and Josep Lloreta ${ }^{1,2}$ \\ ${ }^{1}$ Department of Health and Experimental Sciences, Universitat Pompeu Fabra, Barcelona, Spain; \\ ${ }^{2}$ Department of Pathology, Hospital del Mar, Barcelona, Spain; ${ }^{3}$ Autonomous University of Barcelona, \\ Barcelona, Spain; ${ }^{4}$ Department of Urology, Hospital del Mar, Barcelona, Spain and ${ }^{5}$ Department of \\ Biostatistics, Institut Municipal d'Investigació Mèdica, Hospital del Mar, Barcelona, Spain
}

\begin{abstract}
Prostate cancer is the second cause of cancer-related death in men of the Western World. The role of FGFR3 and its abnormalities in prostate cancer are not known. FGFR3 mutations have been reported in some human tumors. Few studies have analyzed the mutations of FGFR3 in prostate tumors, and no mutations have been previously reported. Prevalence of FGFR3 somatic mutations was investigated in a series of prostate tumors. The presence of other tumors in these patients, including urothelial, skin, colon, and lung neoplasms, was recorded. Mutational analysis of exons 7, 10, and 15 of FGFR3 revealed 9 mutations in the 112 prostate tumors studied $(8 \%)$. Most of them consisted of the missense change S249C. The prevalence of mutations in tumors with combined Gleason score $=6$ is $18 \%(8 / 45)$ compared to $3 \%(1 / 36)$ for tumors with grade $=7$, and $0 \%(0 / 31)$ for those with grade $\geq 8$ and metastases $(P=0.007)$. The frequency of FGFR3 mutations in autopsy and biopsy samples was 6 and $9 \%$, respectively. The prevalence of FGFR3 mutations in prostate tumors from patients with only prostate cancer was $2 \%$ compared to $23 \%$ in prostate tumors from patients with other associated neoplasms $(P=0.001)$. This is the first report of molecular changes of FGFR3 in prostate cancer. This gene does not seem to be central to the pathogenesis of prostate cancer, but it is significantly associated with a subgroup of low-grade prostate tumors, and with the finding of other tumors, mainly arising in bladder and skin. Modern Pathology (2009) 22, 848-856; doi:10.1038/modpathol.2009.46; published online 17 April 2009
\end{abstract}

Keywords: FGFR3; mutation; prostate cancer; bladder cancer; skin tumors

Prostate cancer is the second most common cause of cancer-related death in men of the Western World, and its incidence is increasing. ${ }^{1,2}$ The family of fibroblast growth factors (FGFs) and their receptors are important in prostate organogenesis, ${ }^{3,4}$ and FGFR-MEK-ERK signaling is an integral part of prostate bud induction. ${ }^{5}$ The signaling pathways of FGFs also play prominent roles in prostate biology by mediating stromal-epithelial interactions. ${ }^{3}$ In the prostate, the aberrant signaling of FGFs is a strong factor in the disruption of tissue homeostasis, which, in turn, may contribute to prostate tumor development and progression. ${ }^{6-8}$ FGFs 6 , 8, and 17 are overexpressed in human prostate cancer. In addition, FGF6 expression has also been found to be increased in prostatic intraepithelial

Correspondence: Dr S Hernández, PhD, Department of Pathology, Hospital del Mar, Department of Health and Experimental Sciences, Universitat Pompeu Fabra, Passeig Maritim 25-29, 08003-Barcelona, Spain.

E-mail: silvia.hernandez@upf.edu

Received 15 December 2008; revised and accepted 13 March 2009; published online 17 April 2009 neoplasia. ${ }^{9-11}$ FGF-induced functions are mediated through high-affinity membrane receptor tyrosine kinases. FGF receptors (FGFR1-4) regulate diverse cellular processes, including cell growth, differentiation, and angiogenesis, and they differ in tissue expression, ligand specificity, signal pathway activation, and biological effects. ${ }^{12,13}$ There is a direct causal relationship between the activation of FGFR1 and the angiogenic switch. ${ }^{14}$

In prostate tumors, a differential and changing expression of FGFRs has been observed. FGFR1 and/ or FGFR2 overexpression has been reported in prostate cancer in different papers. However, neither FGFR1 nor FGFR2 expression was found to be statistically related to clinical parameters. ${ }^{15-17}$ Although the overexpression of FGFR1 protein is a common event in both early and late prostate cancer, FGFR4 is increasingly expressed in high-grade tumors. Increased FGFR4 immunoreactivity appears to be significantly associated with decreased patient survival, but not with metastasis. ${ }^{17,18}$

Alterations in FGF signaling regulators also have an impact on prostate tumorigenesis. In this regard, downregulation of Sprouty 1 and Sprouty 2 has been 
recently reported. ${ }^{19-21}$ Although it seems that anomalous FGF signaling is involved in prostate carcinogenesis, the functional role of FGFR3 and its alterations in prostate cancer are not known. FGFR3 protein expression studies show heterogeneous expression levels in benign and malignant prostate epithelium. ${ }^{17,18}$ Gain-of-function mutations have been identified in different dominant autosomal human skeletal disorders such as hypochondroplasia, achondroplasia, and thanatophoric dysplasia. ${ }^{22-24}$ FGFR3 mutations identical to those found in these disorders have been reported in multiple myelomas, cervix and bladder cancer, colon cancer, and benign skin tumors. ${ }^{25-33}$ This gene constitutes a promising marker in the clinical management of patients with low-grade, non-muscle-invasive bladder tumors. ${ }^{29}$ However, very few authors have analyzed the mutational status of the FGFR3 gene in prostate tumors. ${ }^{26,34}$ In this study, we have investigated the prevalence of FGFR3 somatic mutations, as well as the association with the presence of other concurrent neoplasms, in a large group of well-defined prostate tumors classified by combined Gleason score.

\section{Materials and methods}

\section{Patients and Tumor Samples}

A total of 112 prostate tumors, recruited retrospectively from the files of the Department of Pathology, Hospital del Mar, Barcelona, Spain, are the subject of this report. Of these cases, 85 were obtained from biopsy or surgical samples: 25 needle biopsy, 45 radical prostatectomy, and 15 cystoprostatectomy specimens; 18 were tumors found at autopsy, so-called latent tumors. In addition, one lymph node and eight bone metastases were included. To analyze the association of prostate cancer with other tumors, the samples were divided in two groups: a group (G1) with samples from patients with only prostate cancer $(n=82)$ and a multiple tumor group (G2) made up of patients that, in addition to prostate cancer, had other tumors in different locations $(n=30)$. The latter consisted of 18 patients with prostate and bladder tumors; 6 patients with prostate and skin tumors; 2 patients with prostate and colon cancer; 2 patients with prostate, bladder, and skin tumors; 1 patient with prostate and lung cancer; and 1 patient with prostate, bladder, and lung cancer. Among the patients with prostate and bladder cancer, with or without other associated tumors $(n=21), 15$ cases were obtained from cystoprostatectomy specimens and in the remaining 6 cases, prostate cancer samples were obtained from prostatectomy or needle biopsy and bladder tumor samples from transurethral biopsy specimens (Table 1). Based on combined Gleason score, the prostate tumors were classified as grade $=6 \quad(n=45)$, grade $=7 \quad(n=36)$, and grade $\geq 8(n=22)$. Although no information on
Table 1 Summary of the cases according to the different tumor types found in patients

\begin{tabular}{llc}
\hline $\mathrm{N}=112$ & Type of tumors & Number of cases \\
\hline Group $1(n=82)$ & Prostate & 82 \\
Group 2 $(n=30)$ & Prostate and bladder & 18 \\
& Prostate and skin & 6 \\
& Prostate and colon & 2 \\
& Prostate and lung & 1 \\
& Prostate, bladder and skin & 2 \\
& Prostate, bladder and lung & 1 \\
\hline
\end{tabular}

grade was available from the respective primary tumors, metastatic samples $(n=9)$ were grouped together with Gleason score of $\geq 8$, assuming they belonged to an aggressive category. Of the samples, 99 were obtained from formalin-fixed paraffinembedded tumors (FFPE) and 13 samples from frozen tumors. In a subset of 16 cases with prostate and bladder cancer, three types of samples were collected: normal tissue, prostate tumor, and bladder tumor; in 15 of them, the normal and neoplastic prostate tissues was obtained from cystoprostatectomy specimens and in an additional case from needle biopsy. The autopsy samples dated from 1993 to 2006. The remaining cases were recruited between 2000 and 2005. Relevant clinical information was recorded, including age of patients, stage of prostate tumors, basal PSA values, follow-up PSA values, clinical tumor progression, PSA tumor progression, and the presence of previous, simultaneous, or subsequent tumors in other organs.

\section{Cell Lines and Bladder Control Samples}

Two prostate cancer cell lines (PC3 and DU145) and 10 additional superficial (pTa) bladder tumors were also included. The cell lines were obtained from the American Type Culture Collection (Rockville, MD, USA). Theses cases were graded according to both the 2004 WHO and the previous WHO/ISUP systems. Seven tumors were classified as high grade (one grade 3 and six grade 2 cases), and three as low grade (grade 1).

\section{DNA Extraction}

Standard H\&E slides served as templates to select the tumor regions in homologous unstained slides. Areas containing a minimum amount of $>50 \%$, and usually $>90 \%$, tumor cells were manually microdissected from the corresponding sections of FFPE or frozen tissue sections. DNA was extracted from 2-3 consecutive $10 \mu \mathrm{m}$ sections using the Dneasy Tissue Kit (Qiagen GmbH, Hilden, Germany). To avoid the risk of PCR contamination, genomic DNA was extracted in a facility where neither PCR reactions were performed nor PCR products handled. 


\section{DNA Quality Checking}

As most of the autopsy cases were relatively old, we checked the quality of the DNA by performing a previous control PCR. This multiplex PCR reaction involved the amplification of 100, 200, 300, 400, and $600 \mathrm{bp}$ fragments of the $\beta$-actin gene.

\section{FGFR3 PCR Amplification}

FGFR3 exons 7 and 10 were amplified by PCR and sequenced in all samples. In addition, exon 15 amplification and sequencing was performed in 53 tumors. We used exon-specific primers binding to adjacent intron sequences. The primers were: AGTGGCGGTGGTGGTGAGGGAG (forward) and CTGCAAGGTGTACAGTGACGCACA (reverse) for exon 7, CAACGCCCATGTCTTTGCAG (forward) and CAAGATCTCCCGCTTCCCG (reverse) for exon 10, and GAGAGGTGGAGAGGCTTAG (forward) and TCATGCCAGTAGGACGCCT (reverse) for exon 15. PCR reactions were performed in a $25 \mu \mathrm{l}$ volume using 10-50 ng of DNA, $0.2 \mu \mathrm{mol} / \mathrm{l}$ of each primer, $200 \mu \mathrm{mol} / \mathrm{l}$ deoxynucleotide triphosphates, $3.5 \mathrm{mmol} / \mathrm{l} \mathrm{MgCl}_{2}, 1 \times$ PCR II buffer, and 1.5 units of Amplitaq Gold DNA Polymerase (Applied Biosystems, Foster City, CA, USA). PCR conditions were as follows: $95^{\circ} \mathrm{C}(5 \mathrm{~min})$ for 1 cycle, $95^{\circ} \mathrm{C}(40 \mathrm{~s})$, $63^{\circ} \mathrm{C}(40 \mathrm{~s}), 72^{\circ} \mathrm{C}(40 \mathrm{~s})$ for 40 cycles, and a final extension step of $72^{\circ} \mathrm{C}(5 \mathrm{~min})$. PCR products were separated by electrophoresis and visualized with ethidium bromide. Samples without DNA templates were included in all assays as negative controls. PCR products were purified using the Qiagen PCR purification kit (Qiagen, Crawley, United Kingdom) according to the manufacturer's protocol.

\section{FGFR3 Mutation Analysis}

Mutation analysis was done by direct sequencing of purified PCR products using an ABIPRISM 377 instrument and the Big Dye Terminator Kit v.3.1 (Perkin Elmer Applied Biosystems). Each PCR product was sequenced in both forward and reverse directions with the same sets of primers. All sequence changes were confirmed as mutations with two independent rounds of PCR and direct sequencing. As quality control for $20 \%$ of wild-type cases, independent PCR products were amplified and sequenced to confirm the mutational results using DNA from the same tumor area. Ten superficial bladder ( $\mathrm{pTa}$ ) tumor samples were included in the mutational study as controls for the presence of mutations.

\section{Statistical Analysis}

Categorical variables are presented as frequencies and percentages. Fisher's exact test was used to compare categorical variables among groups. A $P$ value of less than 0.05 was considered significant. Statistical analysis was performed using the SPSS statistical package version 12.0 (SPSS Inc., Chicago, IL, USA).

\section{Results}

\section{FGFR3 Mutation Analysis}

The study included 112 prostate tumors, classified according to the revised Gleason system as grade $=6$ $(n=45)$, grade $=7 \quad(n=36)$, and grade $\geq 8(n=22)$. Nine metastatic cases were grouped with the combined Gleason score $\geq 8$ subset. The mutational analysis of exons 7, 10, and 15 of FGFR3 revealed 9 mutations in the 112 prostate cancer samples (8\%). Table 2 summarizes the pathologic features of the mutated cases. The most common mutation was the missense S249C substitution, which was present in seven tumors, followed by A393E, which was found in two tumors.

Less than $3 \%$ of FGFR3 mutations in bladder tumors occur in exon $15,{ }^{35,36}$ however in benign skin tumors such as seborrheic keratoses the prevalence of mutations in the exon 15 (hotspot 652) accounts to about $30 \%$ of the FGFR3 reported mutations. ${ }^{37}$ To test the possibility of finding exon 15 mutations in prostate cancer we screened a subgroup of 53 tumors, classified as grade $=6(n=23)$, grade $=7$ $(n=20)$, grade $\geq 8$, and metastases $(n=10)$. No

Table 2 Spectrum of FGFR3 mutations, polymorphisms, and pathological features of mutated prostate tumors

\begin{tabular}{|c|c|c|c|c|}
\hline Case no. & Tumor precedence & Gleason & Type of alteration & codon \\
\hline 3 & Prostatectomy & $\mathrm{G} 4+3$ & Polymorphism & F386L \\
\hline 7 & Prostatectomy & G4+3 & Polymorphism & F386L \\
\hline 10 & Prostatectomy & $\mathrm{G} 3+3$ & Missense mutation & S249C \\
\hline 23 & Cystoprostatectomy & $\mathrm{G} 3+3$ & Missense mutation & S249C \\
\hline 41 & Prostatectomy & $\mathrm{G} 3+3$ & Missense mutation & S249C \\
\hline 52 & Autopsy & $\mathrm{G} 3+3$ & Missense mutation & S249C \\
\hline 53 & Prostatectomy & $\mathrm{G} 3+3$ & Missense mutation & A393E \\
\hline 77 & Prostatectomy & $\mathrm{G} 3+3$ & Missense mutation & A393E \\
\hline 92 & Cystoprostatectomy & $\mathrm{G} 3+3$ & Missense mutation & S249C \\
\hline 101 & Prostatectomy & $\mathrm{G} 3+3$ & Missense mutation & S249C \\
\hline 103 & Cystoprostatectomy & $\mathrm{G} 4+3$ & Missense mutation & S249C \\
\hline
\end{tabular}


mutation was found in exon 15 in any of these cases. From this subset of tumors, 2 presented the S249C substitution and 51 were wild type for exons 7 and 10.

The two prostate cancer cell lines (PC3 and DU145) were wild type for both exons. To test the sensibility of the technique for detecting mutations, we also performed the mutational analysis on 10 samples of superficial bladder tumors. Four of them $(40 \%)$, classified as low grade $(n=1)$ and high grade-grade $2(n=3)$, presented FGFR3 mutations.

The F386L germline single nucleotide polymorphism (SNP) was detected in two samples ( $2 \%)$, both of them from radical prostatectomy tumors with a combined Gleason score $=7$ (Table 2). The prevalence of this polymorphism in heterozygosity is about 1\% (http://www.ncbi.nlm.nih.gov/SNP).

\section{Distribution of the FGFR3 Mutations According to Combined Gleason Score}

Of the 45 tumors classified as score $=6,8(18 \%)$ and $1(3 \%)$ of the 36 tumors classified as score $=7$ harbored a FGFR3 mutation. Mutations were not found in any of the score $\geq 8$ or the metastatic tumors (Figure 1; Fisher's exact test, $P=0.007$ ). Taking all the cases with combined Gleason score $\geq 7$ and the metastases together, the percentage of mutations was $1.5 \%$ ( 1 of 67 cases), and the difference with combined Gleason score $=6$ cases was also statistically significant (Fisher's exact test, $P=0.003$ )

\section{Distribution of the FGFR3 Mutations According to Sample Origin}

According to their origin, 85 cases were biopsy (needle biopsy, radical prostatectomy, or cystoprostatectomy tumors), 18 were tumors found at autopsy (latent tumors), and 9 samples were prostate cancer

Prevalence of FGFR3 mutations according to the Gleason grade classification

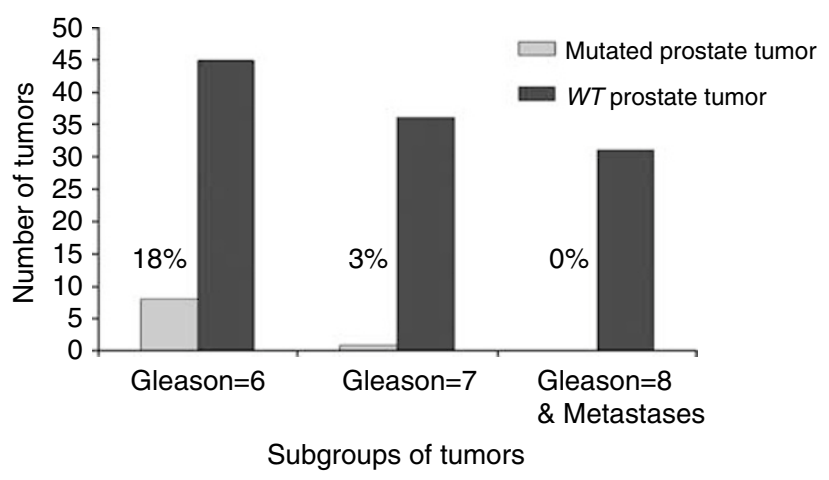

Figure 1 Prevalence of FGFR3 mutations in the different tumor combined Gleason scores. 18\% of tumors classified as Gleason score $=6$ and $3 \%$ of tumors classified as Gleason score $=7$ harbored a FGFR3 mutation (Fisher's exact test, $P=0.007$ ). metastases. Eight mutations were found in the 85 biopsy samples (9\%); we detected a S249C mutation in one of the 18 autopsy cases $(6 \%)$ whereas none of the metastatic samples harbored FGFR3 mutations.

\section{Relationship between FGFR3 Status and Biochemical Recurrence}

Of the 81 patients with available PSA data, biochemical recurrence was detected in 19, whereas in the remaining 62 cases there was no sign of biochemical or clinical recurrence at last follow-up. Of the cases, 8 presented FGFR3 mutation and 73 were wild type. There was no association between the presence of FGFR3 mutations and biochemical recurrence. However, there was a significant association between combined Gleason score and biochemical recurrence, as about $70 \%$ of Gleason score $\geq 8$ and metastatic patients developed biochemical recurrence, whereas only $12 \%$ of Gleason score $=6$ and $10 \%$ of Gleason score $=7$ patients did recur (Fisher's exact test $P=0.0001$ ). These results are an indication that this series of patients was not biased and reflected the normal spectrum of the disease. When examining the possible association between specific mutations and biochemical recurrence, none of the six cases with the S249C mutation recurred, whereas the two cases with the A393E mutation did recur. Although this could reflect a real trend, the number of cases is too low to draw any definitive conclusions in this regard.

\section{Distribution of the FGFR3 Mutations in Cases with and without Other Associated Tumors}

Taking into account the number of different tumor types, the cases were classified as group 1 (G1) when only prostate cancer was found $(n=82)$, and group 2 (G2) when, in addition to prostate cancer, there were other neoplasms in different organs $(n=30)$. In G2, 18 patients had prostate and bladder tumors, 6 patients had prostate and skin tumors, 2 patients presented with prostate and colon cancer, 2 patients with prostate, bladder, and skin tumors, 1 patient had prostate and lung cancer, and 1 patient prostate, bladder, and lung cancer (Table 1). The clinicopathologic features of all cases from G2 are summarized in Table 3.

FGFR 3 mutations were found in only 2 of $82(2 \%)$ cases from G1. In contrast, FGFR3 mutations were present in 7 of $30(23 \%)$ prostate cancer samples from G2 (Figure 2). This difference was statistically significant (Fisher's exact test, $P=0.001$ ). All the mutated cases from G2 had prostate cancer associated with only one additional neoplasm. Moreover, analyzing different tumor combinations in G2, 3 of $18(17 \%)$ patients with only prostate and bladder cancer harbored a FGFR3 mutation in the prostate tumor. In the subgroup with only prostate and skin tumors, three of six $(50 \%)$ prostate cancer 
Table 3 FGFR3 mutation analysis in specimens of the mixed tumor group (G2)

\begin{tabular}{|c|c|c|c|c|c|c|}
\hline \multirow[b]{2}{*}{ Case no. } & \multirow[b]{2}{*}{ Sample type } & \multicolumn{5}{|c|}{ Mixed tumor group (G2) } \\
\hline & & $\begin{array}{l}\text { FGFR3 status in } \\
\text { prostate tumor }\end{array}$ & Gleason & Other tumors & $\begin{array}{l}\text { FGFR3 status in } \\
\text { other tumors }\end{array}$ & $\begin{array}{l}\text { FGFR3 status in } \\
\text { normal prostate }\end{array}$ \\
\hline 14 & Cystoprostatectomy & WT & $\mathrm{G} 3+4$ & Bladder T4G3 & WT & WT \\
\hline 21 & Cystoprostatectomy & WT & $\mathrm{G} 3+4$ & Bladder T2G2 & S249C & WT \\
\hline 25 & Cystoprostatectomy & WT & $\mathrm{G} 3+3$ & Bladder T4G3 & WT & WT \\
\hline 23 & Prostatectomy & S249C & $\mathrm{G} 3+3$ & Bladder TaG1 & WT & WT \\
\hline 27 & Cystoprostatectomy & WT & $\mathrm{G} 3+3$ & Bladder T2G3 & WT & WT \\
\hline 37 & Cystoprostatectomy & WT & $\mathrm{G} 3+3$ & Bladder T4G3 & WT & WT \\
\hline 43 & Cystoprostatectomy & WT & $\mathrm{G} 3+3$ & Bladder T1G3/lung cancer & WT/not done & WT \\
\hline 50 & Cystoprostatectomy & WT & $\mathrm{G} 3+3$ & Bladder T2G3 & WT & WT \\
\hline 56 & Cystoprostatectomy & WT & $\mathrm{G} 3+3$ & Bladder T3G3 & WT & WT \\
\hline 83 & Cystoprostatectomy & WT & $\mathrm{G} 3+3$ & Bladder TaG3 & WT & WT \\
\hline 85 & Cystoprostatectomy & WT & $\mathrm{G} 4+5$ & $\begin{array}{l}\text { Bladder T4G3/squamous } \\
\text { carcinoma skin }\end{array}$ & WT/not done & WT \\
\hline 88 & Cystoprostatectomy & WT & $\mathrm{G} 3+3$ & Bladder TaG3 & WT & WT \\
\hline 92 & Cystoprostatectomy & S249C & $\mathrm{G} 3+3$ & Bladder T2G3 & WT & WT \\
\hline 103 & Cystoprostatectomy & S249C & G4+3 & Bladder T4G3 & WT & WT \\
\hline 104 & Cystoprostatectomy & WT & $\mathrm{G} 3+3$ & $\begin{array}{l}\text { Bladder T2G3/actinic } \\
\text { keratosis }\end{array}$ & WT/not done & WT \\
\hline 108 & Cystoprostatectomy & WT & $\mathrm{G} 3+3$ & Bladder T3G3 & WT & WT \\
\hline 26 & Needle biopsy & WT & $\mathrm{G} 3+4$ & Bladder TaG2 & Not done & Not done \\
\hline 54 & Needle biopsy & WT & $\mathrm{G} 4+4$ & Bladder TaG2 & Not done & Not done \\
\hline 63 & Prostatectomy & WT & $\mathrm{G} 3+4$ & Bladder T1G3 & Not done & Not done \\
\hline 84 & Needle biopsy & WT & $\mathrm{G} 3+3$ & Bladder TaG2 & Not done & Not done \\
\hline 100 & Prostatectomy & WT & $\mathrm{G} 4+3$ & Bladder TaG2 & Not done & Not done \\
\hline 28 & Metastasis & WT & - & $\begin{array}{l}\text { Squamous carcinoma } \\
\text { skin/actinic keratosis }\end{array}$ & Not done & Not done \\
\hline 41 & Prostatectomy & S249C & $\mathrm{G} 3+3$ & Basal cell carcinoma & Not done & Not done \\
\hline 53 & Prostatectomy & A393E & $\mathrm{G} 3+3$ & Seborrheic keratosis & Not done & Not done \\
\hline 68 & Needle Biopsy & WT & $\mathrm{G} 4+4$ & Seborrheic keratosis & Not done & Not done \\
\hline 76 & Needle Biopsy & WT & $\mathrm{G} 4+3$ & Seborrheic keratosis & Not done & Not done \\
\hline 77 & Prostatectomy & A393E & $\mathrm{G} 3+3$ & Seborrheic keratosis & Not done & Not done \\
\hline 10 & Prostatectomy & S249C & $\mathrm{G} 3+3$ & Colon cancer & Not done & Not done \\
\hline 40 & Prostatectomy & WT & $\mathrm{G} 3+4$ & Lung cancer & Not done & Not done \\
\hline 44 & Prostatectomy & WT & $\mathrm{G} 3+3$ & Colon cancer & Not done & Not done \\
\hline
\end{tabular}

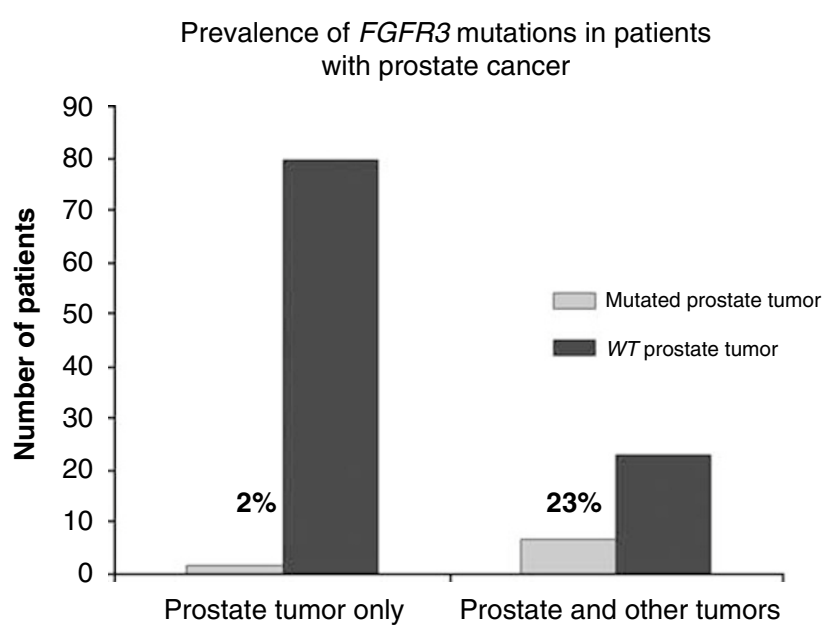

Figure 2 Prevalence of FGFR3 mutations in the different tumor prostate groups according the presence of another type of cancer. The prevalence of FGFR3 mutations in patients with only prostate tumors was 2 vs $23 \%$ in patients with prostate and other types of tumors (Fisher's exact test, $P=0.001$ ). samples presented a FGFR3 mutation. Finally, one case with only prostate and colon cancer also had a FGFR3 mutation. The two cases with prostate, bladder, and skin tumors, and the case with prostate and lung cancer were wild type for FGFR3, in the analysis of exons 7 and 10 (Figure 3). Comparing G1 to the subset of G2 cases with only prostate and bladder cancer, the difference in FGFR3 mutation frequency ( 2 vs $17 \%$ ) was also statistically significant (Fisher's exact Test, $P=0.039$ ). The mutational status of all G2 cases is also summarized in Table 3.

In a subset of patients from G2, including all the cystoprostatectomy cases $(n=15)$ and an additional case with prostate and bladder biopsies, we also studied the presence of FGFR3 mutations in the corresponding normal prostate and bladder tumors. All the normal prostate tissue samples were wild type for exons 7 and 10. Only one bladder tumor presented a S249C mutation (1 of $16 ; 6 \%$ ), and in this case the corresponding prostate tumor and normal prostate tissue were wild type (Table 3 ). 
Prevalence of FGFR3 mutations in patients with different combinations of prostate and other tumors

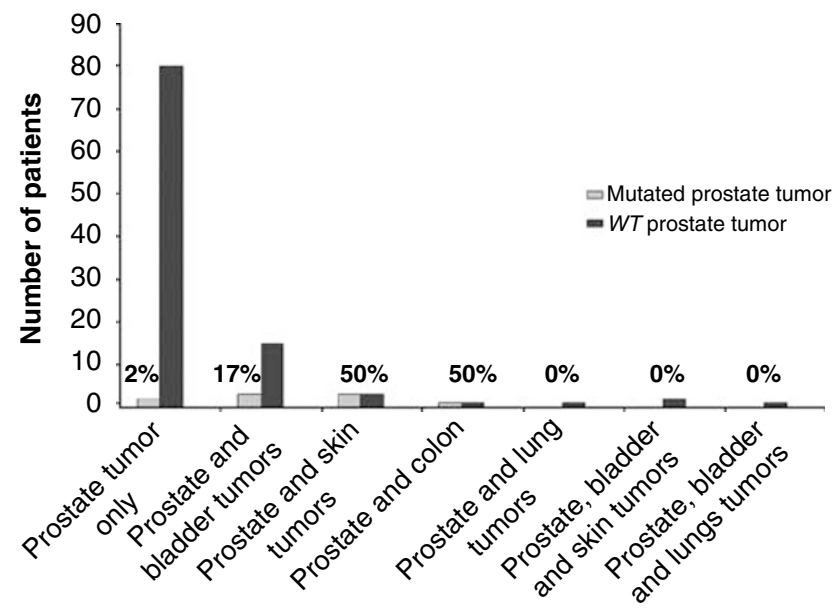

Figure 3 Prevalence of FGFR3 mutations in the different combinations of prostate cancer and another type of tumors. The prevalence of FGFR3 mutations in the cases with prostate and bladder tumors $(17 \%)$ is significantly higher than in patients with only prostate cancer (Fisher's exact test, $P=0.039$ ).

\section{Discussion}

Prostate cancer is the most common cancer in men in Europe and the second cause of cancer-related death in men of the Western World. Its incidence is increasing, but progression rate is only about $30 \% .^{1,2}$ More refined detection methods are increasing the number of patients that undergo radical treatment for tumors that perhaps would not progress or that would eventually progress after many years, thus resulting in increased survival and also higher morbidity rates. ${ }^{38}$ Due to the difficulties in obtaining samples for high throughput molecular analysis from early tumors, genes that could be decisive for the transition from a latent status to an aggressive phase leading to progression are still unknown. Therefore, there are no clues to select patients for a less radical treatment or for a safe watchful waiting.

Many different genes have been investigated for their potential roles in the development and progression of prostate cancer. $^{39,40}$ However, in the present time there are no relevant molecular markers associated with tumor progression, with the different subsets of prostate cancer or with some of the clinicopathological features of this type of tumor. There is a need to better identify patients who have a low risk of progression, to avoid overtreating them, as well as those who are likely to progress to treat them more aggressively.

FGFR3 molecular alterations seem to be restricted to a few types of human cancer. Mutations in this gene have been reported in bladder and cervix carcinoma, multiple myeloma, colon cancer and, more recently, in benign skin tumors. ${ }^{25-33}$ The relatively high prevalence of FGFR3 mutations in benign skin tumors and in noninvasive bladder tumors suggests an association of FGFR3 mutation with low risk cancers. ${ }^{35,41}$ Although it seems that anomalous FGF signaling is involved in prostate carcinogenesis, the functional role of FGFR3 and its alterations in prostate cancer are unknown.

The purpose of this study was to investigate the prevalence of FGFR3 somatic mutations in a large group of well-defined prostate tumors classified by stage and combined Gleason score. This is the first report of the presence of FGFR3 genetic alterations in prostate cancer. The mutational analysis revealed that the FGFR3 gene is mutated in a small proportion $(8 \%)$ of prostate tumors.

Nevertheless, if we grouped the cases according to the combined Gleason score, the presence of FGFR3 abnormalities was associated with less aggressive prostate tumors, as $89 \%$ of mutations ( 8 out of 9 ) were found in tumors with a combined Gleason score $=6 ; 100 \%$ of mutations were present in tumors with Gleason score $\leq 7$ and, consequently, none of the Gleason score $\geq 8$ or the metastatic tumors harbored any mutations. Comparing all cases with combined Gleason score $=6$ with those with combined Gleason score $>6$ and metastases taken together, the presence of mutations in the former was statistically significant. Thus, we can conclude that mutations of FGFR3 are associated with a subset of prostate tumors with low combined Gleason score.

Only two previous papers have analyzed the FGFR3 mutational status in prostate cancer, all of them providing negative results. ${ }^{26,34}$ Sibley et $a l^{26}$ analyzed the mutational status of exons 7,10 , and 15 in only 10 samples, as well as in prostate cancer cell lines DU145, PC3, and LNCap. In concordance with our study, DU145 and PC3 were wild type for FGFR3. They did not find FGFR3 mutations in other tumors included in their series, such as stomach, colon, ovary, brain, and kidney cancers. In most of these subgroups, the number of samples was too low to allow any definitive conclusions on the mutational status of FGFR3. In a subsequent paper, Jang et $a l^{33}$ reported the presence of FGFR3 somatic mutations in about $5 \%$ of colon tumors in a series of 40 cases.

Naimi et $a l^{34}$ investigated the mutational status of the junction between the Ig domains II and III of FGFR3, which included exon 7 of the gene, in 20 prostate tumors. In this study, no mutation was detected. According to our findings, the prevalence of mutations in Gleason score $\leq 7$ cases is about $11 \%$, and most of them were Gleason score $=6$. In the Naimi et al study, only 14 tumors with Gleason score $\leq 7$ were included, so their sample size approached the threshold for detection.

In summary, the vast majority of prostate tumors with a FGFR3 mutation were low-grade tumors. Similar to what has been reported in bladder cancer, the alterations of this gene in prostate cancer appear to be associated with the less aggressive prostatic tumors. These findings are in keeping with the previously reported conception that FGFR3 
mutations are more prevalent in low-grade or benign neoplasms. ${ }^{35,41}$

According to the clinicopathological setting of the tumors, the prevalence of mutations in the autopsy (latent) tumors was 6\% and in the biopsy samples $9 \%$. In that sense, this gene does not seem to be selectively altered in the group of tumors found at autopsy, which theoretically would be mostly made up of low-grade, slowly growing tumors.

The review of the clinicopathological data of the patients revealed that some of them presented, in addition to the prostate cancer, other simultaneous or previous neoplasms in other sites. The other lesions identified in these patients were mostly urothelial and benign skin tumors, and there were also few cases associated with colon and lung tumors. The results of the mutational study were then compared in two subgroups: a subgroup of cases with only prostate cancer, G1, and another subgroup with prostate cancer and an additional different neoplasm, G2 (bladder, skin, colon, and lung). Comparing the FGFR3 status between these two groups, the presence of FGFR3 mutations was significantly more prevalent in G2. Of patients with prostate cancer and other tumors, $23 \%$ harbored a FGFR3 mutation compared to $2 \%$ of FGFR3 mutations in patients with prostate cancer only. When separately analyzing the different tumor combinations in G2, the prevalence of FGFR3 mutations in patients with prostate and bladder tumors was much higher $(17 \%)$ than that of patients with only prostate tumors $(2 \%)$. Furthermore, $50 \%$ of cases with prostate and skin tumors harbored a FGFR3 mutation in the prostate tumor. Finally, one case with prostate and colon cancer had a mutation. Therefore, it is possible to conclude that FGFR3 mutations are more prevalent in a subset of prostate tumors from patients with concurrent bladder cancer. On the other hand, although the number of cases with both prostate and skin tumors is very low in our series, the coexistence of these two neoplasms seems to be strongly related with FGFR3 mutations in the respective prostate carcinomas.

As FGFR3 is a gene involved in bladder carcinogenesis and the presence of FGFR3 mutations is also a common event in benign skin tumors, ${ }^{30-32,35,37,41}$ this association between bladder, skin, and prostate cancer could suggest a common role for FGFR3 in their pathogenesis. However, the mutational analysis of the corresponding bladder tumors and normal prostate samples in some of the patients from our study revealed the wild-type status of the gene. In fact, if the mutation did not occur in the germline, the normal tissues should not harbor it. The lack of FGFR3 mutations in the bladder tumors from the patients with FGFR3 mutated prostate tumors could indicate that the two tumors are unrelated. However, it should be noted that most of the bladder tumors investigated in the mixed tumor group belonged to a group of invasive muscle bladder cancers. It is well known that FGFR3 mutations are frequent in superficial tumors but not in muscle-invasive tumors. ${ }^{27-29,35}$ Thus, another possible explanation for the discrepant mutational status of FGFR3 in the bladder and prostate tumors could be that the bladder tumor had lost the mutated FGFR3 allele along its progression.

The relationship between prostate and skin tumors has not yet been previously investigated. The finding that $50 \%$ of patients with prostate and skin tumors in our series harbored FGFR3 mutations seems to indicate that this gene could be related with the pathogenesis not only of concurrent bladder and skin tumors, but also of the concurrent prostate and skin tumors. Interestingly, none of the two cases with prostate, bladder, and skin tumors harbored FGFR3 mutations.

A possible association between FGFR3 mutations and tumors in different organs has been postulated by different authors. ${ }^{32,41}$ The fact that a high proportion of low-grade urothelial carcinomas and benign skin tumors harbors FGFR3 mutations suggested a potential involvement of these mutations in the pathogenesis of both entities. Furthermore, some patients with epidermal nevi developed an urothelial carcinoma at an early age. Nevertheless, neither the bladder tumors nor the epidermal nevi in these previous series displayed FGFR3 mutations, and the authors suggested that other genes may be involved in the pathogenesis of both tumors in these patients or that FGFR3 mutations could involve other exons. ${ }^{32,41}$ Our findings in prostate cancer have lead us to speculate with an association between FGFR3 mutations and presence of tumors in different organs, specially bladder and/or benign skin tumors. This not being a germline alteration, it can be speculated that mutations could involve selectively different tissues in the same patients. In this regard, previous studies in epidermal nevi revealed that FGFR3 somatic mutations can occur in mosaicism. Skin mosaicism results when postzygotic mutations lead to the presence of two or more genetically different cell populations in the same individual. ${ }^{42}$ It has been shown that a proportion of epidermal nevi result from activating embryonic mutations in FGFR3 and PI3K presenting with mosaicism in the skin. ${ }^{32,43,44}$

In any event, similar to what has been reported for bladder and skin tumors, the association of FGFR3 alterations with prostate and skin tumors cannot be firmly established from the results of this study, and it deserves further research in larger series of patients, to elucidate the role of FGFR3 in the pathogenesis of different coexisting tumors. The cell type affected by the FGFR3 mutation as well as different involved cofactors and signaling pathways may contribute to the diverse tumoral phenotypes. ${ }^{31}$

Another interesting finding was the identification of a FGFR3 mutation in the prostate tumor from one of the two cases with a simultaneous colon cancer. Mutations in this gene have been previously identified in colorectal cancer by Jang et $a l,{ }^{33}$ and 
it could be interesting to investigate the possible relationship between the two tumors in a larger cohort of patients.

With regard to the type of mutation, a wide range of somatic hotspot mutations have been described in low-grade urothelial carcinomas and in SK, whereas the majority of EN display the R248C substitution. $^{35,41}$ The most common mutation found in our series of prostate tumors was S249C, whereas A393E was present in two tumors. The S249C substitution is the most frequent mutation described in bladder cancer and the only one found to date in cervical carcinomas. A393E is a rare mutation in bladder cancer, about $1 \%$ of all reported mutations. However, it has been associated with bladder tumors classified as low malignant potential neoplasms. ${ }^{25,29,35}$ In SK, the A393E substitution has been reported with a frequency of about $9 \%$ of the adenoid SK harboring FGFR3 mutations. ${ }^{45}$

In sharp contrast with the inhibitory role of the mutated forms of the FGFR3 gene in bone growth, FGFR3 mutations have been suggested to play an oncogenic role in human tumors. ${ }^{46}$ However, the exact mechanism by which mutated FGFR3b is involved in epithelial tumor progression is not well understood. Moreover, the suggested oncogenic role of mutated FGFR3 in different types of tumors is controversial, with a striking high prevalence of mutated benign skin tumors. In the S249C substitution, the newly created cysteine leads to the formation of bisulfite bonds between the extracellular domains of the two FGF receptors and to ligand-independent constitutive receptor activation. On the other hand, the A393E mutation increases the fraction of FGFR3 dimers resulting from dimer stabilization, measured by the change in the free energy of dimerization. ${ }^{35,47}$ In addition, BernardPierrot et $a l^{46}$ demonstrated that FGFR3b-S249C was able to transform NIH-3T3 cells, inducing their anchorage-independent growth and tumor transformation when injected subcutaneously into nude mice, with the transforming properties classically attributed to oncogenes.

In conclusion, the results of this study suggest that although FGFR3 does not seem to be central in the pathogenesis of prostate cancer, it is associated with a subpopulation of low-grade prostate tumors, and also with a subset of prostate carcinomas found incidentally in patients with concurrent bladder cancer or skin tumors. More studies are needed to clarify whether FGFR3 alterations are useful to identity patients with a low risk of progression, and to investigate the possible relationship between FGFR3 and the presence of concurrent tumors in the same patients.

\section{Acknowledgement}

Grants FIS/Instituto de Salud Carlos III 965190005 and 06/1411, made by the Ministry of Health of the
Spanish Government, Support Grant 2006 and Support Grant 2008 from the Spanish Association Against Cancer (Barcelona Territorial Board).

\section{References}

1 Jemal A, Siegel R, Ward E, et al. Cancer statistics, 2007. CA Cancer J Clin 2007;57:43-66.

2 Woolf SH. Screening for prostate cancer with prostatespecific antigen. An examination of the evidence. $\mathrm{N}$ Engl J Med 1995;33:1401-1405.

3 Thomson A. Role of androgens and fibroblast growth factors in prostatic development. Reproduction 2001;121:187-195.

4 Lin Y, Liu G, Zhang Y, et al. Fibroblast growth factor receptor 2 tyrosine kinase is required for prostatic morphogenesis and the acquisition of strict androgen dependency for adult tissue homeostasis. Development 2007;134:723-734.

5 Kuslak SL, Marker PC. Fibroblast growth factor receptor signaling through MEK-ERK is required for prostate bud induction. Differentiation 2007;75: 638-651.

6 Jin C, McKeehan K, Guo W, et al. Cooperation between ectopic FGFR1 and depression of FGFR2 in induction of prostatic intraepithelial neoplasia in the mouse prostate. Cancer Res 2003;63:8784-8790.

7 Jin C, Wang F, Wu X, et al. Directionally specific paracrine communication mediated by epithelial FGF9 to stromal FGFR3 in two-compartment premalignant prostate tumors. Cancer Res 2004;64:4555-4562.

$8 \mathrm{Wu}$ X, Jin C, Wang F, et al. Stromal cell heterogeneity in fibroblast growth factor-mediated stromal-epithelial cell cross-talk in premalignant prostate tumors. Cancer Res 2003;63:4936-4944.

9 Heer R, Douglas D, Mathers ME, et al. Fibroblast growth factor 17 is over-expressed in human prostate cancer. J Pathol 2004;204:578-586.

10 Ropiquet F, Giri D, Kwabi-Addo B, et al. Increased expression of fibroblast growth factor 6 in human prostatic intraepithelial neoplasia and prostate cancer. Cancer Res 2000;60:4245-4250.

11 Tanaka A, Furuya A, Yamasaki M, et al. High frequency of fibroblast growth factor (FGF) 8 expression in clinical prostate cancers and breast tissues, immunohistochemically demonstrated by a newly established neutralizing monoclonal antibody against FGF 8. Cancer Res 1998;58:2053-2056.

12 Powers CJ, McLeskey SW, Wellstein A. Fibroblast growth factors, their receptors and signaling. Endocr Relat Cancer 2000;7:165-197.

13 Shaoul E, Reich-Slotky R, Berman B, et al. Fibroblast growth factor receptors display both common and distinct signaling pathways. Oncogene 1995;10: 1553-1561.

14 Winter SF, Acevedo VD, Gangula RD, et al. Conditional activation of FGFR1 in the prostate epithelium induces angiogenesis with concomitant differential regulation of Ang-1 and Ang-2. Oncogene 2007;26:4897-4907.

15 Giri D, Ropiquet F, Ittmann M. Alterations in expression of basic fibroblast growth factor (FGF) 2 and its receptor FGFR-1 in human prostate cancer. Clin Cancer Res 1999;5:1063-1071.

16 Valve EM, Nevalainen MT, Nurmi MJ, et al. Increased expression of FGF-8 isoforms and FGF receptors in 
human premalignant prostatic intraepithelial neoplasia lesions and prostate cancer. Lab Invest 2001;81: 815-826.

17 Sahadevan K, Darby S, Leung HY, et al. Selective overexpression of fibroblast growth factor receptors 1 and 4 in clinical prostate cancer. J Pathol 2007;213:82-90.

18 Gowardhan B, Douglas DA, Mathers ME, et al. Evaluation of the fibroblast growth factor system as a potential target for therapy in human prostate cancer. Br J Cancer 2005;92:320-327.

19 Fritzsche S, Kenzelmann M, Hoffmann MJ, et al. Concomitant down-regulation of SPRY1 and SPRY2 in prostate carcinoma. Endocr Relat Cancer 2006;13: 839-849.

20 Kwabi-Addo B, Wang J, Erdem H, et al. The expression of Sprouty1, an inhibitor of fibroblast growth factor signal transduction, is decreased in human prostate cancer. Cancer Res 2004;64:4728-4735.

21 McKie AB, Douglas DA, Olijslagers SY, et al. Epigenetic inactivation of the human sprouty2 (hSPRY2) homologue in prostate cancer. Oncogene 2005;24: 2166-2174

22 Horton WA, Lunstrum GP. Fibroblast growth factor receptor 3 mutations in achondroplasia and related forms of dwarfism. Rev Endocr Metab Disord 2002;3: 381-385.

23 Ornitz DM, Marie PJ. FGF signaling pathways in endochondral and intramembranous bone development and human genetic disease. Genes Dev 2002;16:1446-1465.

24 Ornitz DM. FGF signaling in the developing endochondral skeleton. Cytokine Growth Factor Rev 2005;16:205-213.

25 Cappellen D, De Oliveira C, Ricol D, et al. Frequent activating mutations of FGFR3 in human bladder and cervix carcinomas. Nat Genet 1999;23:18-20.

26 Sibley K, Stern P, Knowles MA. Frequency of fibroblast growth factor receptor 3 mutations in sporadic tumors. Oncogene 2001;20:4416-4418.

27 Billerey C, Chopin D, Aubriot-Lorton $\mathrm{MH}$, et al. Frequent FGFR3 mutations in papillary non-invasive bladder (pTa) tumors. Am J Pathol 2001;158: 1955-1959.

28 Hernandez S, Lopez-Knowles E, Lloreta J, et al. FGFR3 and Tp53 mutations in T1G3 transitional bladder carcinomas: independent distribution and lack of association with prognosis. Clin Cancer Res 2005;11: 5444-5450.

29 Hernandez S, Lopez-Knowles E, Lloreta J, et al. Prospective study of FGFR3 mutations as a prognostic factor in nonmuscle invasive urothelial bladder carcinomas. J Clin Oncol 2006;24:3664-3671.

30 Logie A, Dunois-Larde C, Rosty C, et al. Activating mutations of the tyrosine kinase receptor FGFR3 are associated with benign skin tumors in mice and humans. Hum Mol Genet 2005;14:1153-1160.

31 Hafner C, Vogt T, Hartmann A. FGFR3 mutations in benign skin tumors. Cell Cycle 2006;5:2723-2728.
32 Hernandez S, Toll A, Baselga E, et al. Fibroblast growth factor receptor 3 mutations in epidermal nevi and associated low grade bladder tumors. J Invest Dermatol 2007;127:1664-1666.

33 Jang JH, Shin KH, Park JG. Mutations in fibroblast growth factor receptor 2 and fibroblast growth factor receptor 3 genes associated with human gastric and colorectal cancers. Cancer Res 2001;61:3541-3543.

34 Naimi B, Latil A, Berthon P, et al. No evidence for fibroblast growth factor receptor 3 (FGFR-3) R248C/ S249C mutations in human prostate cancer. Int J Cancer 2000;87:455-456.

35 Knowles MA. Role of FGFR3 in urotelial cell carcinoma: biomarker and potencial therapeutic target. World J Urol 2007;25:581-593.

36 Hafner C, Hartmann A, van Oers JM, et al. FGFR3 mutations in seborrheic keratoses are already present in flat lesions and associated with age and localization. Mod Pathol 2007;20:895-903.

37 van Rhijn BW, Vis AN, van der Kwast $\mathrm{TH}$, et al. Molecular grading of urothelial cell carcinoma with fibroblast growth factor receptor 3 and MIB-1 is superior to pathologic grade for the prediction of clinical outcome. J Clin Oncol 2003;21:1912-1921.

38 Damber JE, Aus G. Prostate cancer. Lancet 2008;371:1710-1721.

39 Reynolds MA, Kastury K, Groskopf J, et al. Molecular markers for prostate cancer. Cancer Lett 2007;249: 5-13.

40 Kopper L, Tímár J. Genomics of prostate cancer: is there anything to 'translate'? Pathol Oncol Res 2005; 11:197-203.

41 Hafner C, Hartmann A, Vogt T. FGFR3 mutations in epidermal nevi and seborrheic keratoses: lessons from Urothelium and skin. J Invest Dermatol 2007;127:1572-1573.

42 Toll A, Real F. Somatic oncogenic mutations, benign skin lesions and cancer progression: where to look next? Cell Cycle 2008;7:2674-2681.

43 Christian Hafner C, van Oers JM, Vogt $\mathrm{T}$, et al. Mosaicism of activatingFGFR3 mutations in human skin causes epidermal nevi. J Clin Invest 2006;116: 2201-2207.

44 Hafner C, López-Knowles E, Nuno ML, et al. Oncogenic PIK3CA mutations occur in epidermal nevi and seborrheic keratoses with a characteristic mutation pattern. Proc Natl Acad Sci USA 2007;104: 13450-13454.

45 Hafner C, van Oers JM, Hartmann A, et al. High frequency of FGFR3 mutations in adenoid seborrheic keratoses. J Invest Dermatol 2006;126:2404-2407.

46 Bernard-Pierrot I, Brams A, Dunois-Lardé C, et al. Oncogenic properties of the mutated forms of fibroblast growth factor receptor $3 \mathrm{~b}$. Carcinogenesis 2006;27:740-747.

47 Li E, You M, Hristova K. FGFR3 dimer stabilization due to a single amino acid pathogenic mutation. J Mol Biol 2006;356:600-612. 